\title{
Optimal Scintigraphic Evaluation of a Hydronephrotic Horseshoe Kidney
}

\author{
Mark L. Lessne, Nirav P. Shah, James F. Stinchon, Mary E. Cross, CNMT, and M. Elizabeth Oates \\ Nuclear Medicine Section, Department of Radiology, Boston University Medical Center, Boston, Massachusetts
}

\begin{abstract}
A 25-y-old man with horseshoe kidney was referred for diureticaugmented renal scintigraphy. Single-detector dynamic posterior imaging was performed and revealed asymmetric retention of radiotracer in the left collecting system. Renal scintigraphy was repeated with a modified protocol. Dynamic imaging was performed this time using dual-detector acquisition of both anterior and posterior data. Thereafter, pregravity and subsequently postgravity static images were obtained in both anterior and posterior projections. This second study showed near-complete emptying of the left collecting system. This case illustrates the utility of using simultaneous anterior and posterior imaging and geometric mean calculations for functional analysis and also highlights the value of physiologic maneuvers to augment the traditional diuretic challenge.
\end{abstract}

Key Words: diuretic renal scintigraphy; horseshoe kidney; gravity-assisted urinary drainage; equivocal urinary tract obstruction

J Nucl Med Technol 2007; 35:255-258

DOI: $10.2967 /$ jnmt.106.034462

$\mathbf{T}$ he horseshoe kidney is the most common renal fusion anomaly, with an estimated incidence of 1 in 400 persons (1). The anomaly consists of 2 renal masses connected at their lower poles by a parenchymal or fibrous isthmus. Several hypotheses have attempted to explain the malformation, including attribution of the fusion anomaly to a converging course of the ureteric buds, a medial position of the metanephric ducts relative to the umbilical arteries, or some combination thereof (2). Though one third of patients remain asymptomatic, nonspecific abdominal pain radiating to the lower back is a common complaint (1). Ureteropelvic junction obstruction occurs in up to one third of patients with the anomaly (1). More common is hydronephrosis or hydroureteronephrosis, which may be secondary either to a mechanical obstruction or to functional dilatation due to atonicity of renal pelvic and ureteral tissue (3). Therefore, further studies are often required to determine the presence and degree of obstruction and its impact on renal function.

\footnotetext{
Received Jun. 30, 2006; revision accepted Jul. 3, 2007.

For correspondence or reprints contact: Nirav P. Shah, MD, Charlotte Radiology, Carolinas Healthcare System, 1701 East Blvd., Charlotte, NC 28203.

E-mail: Nirav.Shah@charlotteradiology.com

COPYRIGHT @ 2007 by the Society of Nuclear Medicine, Inc.
}

Renal ultrasound or CT is often performed to define the morphology of a horseshoe kidney, evaluate its vascular supply and urinary drainage, and assess for possible causes of obstruction, such as stone disease or coexistent mass lesions. Renal scintigraphy using ${ }^{99 \mathrm{~m}} \mathrm{Tc}$-mercaptoacetyltriglycine ( $\left.{ }^{99 \mathrm{~m}} \mathrm{Tc}-\mathrm{MAG} 3\right)$ allows for characterization of renal perfusion, function, excretion and, to some extent, shows the morphology of the upper urinary tract (4). Traditionally, posterior imaging is obtained after ${ }^{99} \mathrm{~m}$ Tc-MAG3 injection because the usual kidney position is in the posterior upper abdomen. If mechanical obstruction remains a consideration based on visual inspection and time-activity curve analysis, intravenous furosemide is administered "as soon as good tracer accumulation within the dilated pelvicalyceal system has occurred, followed by additional imaging" (5). The application of diuretic-augmented renal scintigraphy in addition to baseline imaging has found widespread use in the differentiation of a mechanical from a functional cause of a dilated upper tract, and the protocol and acquisition standards for these studies in evaluating normally located kidneys are well described (6).

We present a case of a horseshoe kidney with hydronephrosis and hydroureter evaluated by diuresis renal scintigraphy. This case illustrates the utility of using simultaneous anterior and posterior imaging and geometric mean calculations for functional analysis and also highlights the value of physiologic maneuvers to augment the traditional diuretic challenge.

\section{CASE REPORT}

A 25-y-old man presented to his primary care physician with an incidental finding of left hydroureter and hydronephrosis on ultrasound. He had a remote history of culturenegative dysuria, unrelated to increased fluid or alcohol intake, but otherwise his medical history was noncontributory. A CT scan with intravenous contrast medium revealed a horseshoe kidney, with severe hydronephrosis of the left moiety and significant dilatation of the left renal pelvis at the proximal ureter (Fig. 1). No definite etiology for this enlargement was found on CT examination. The right moiety was normal.

Diuretic-augmented renal scintigraphy was performed with a standard protocol to evaluate renal function and establish the significance of the dilated left collecting 
FIGURE 1. Axial cranial (A) to caudal (B) CT images demonstrate horseshoe kidney with dilated, hydronephrotic left collecting system.
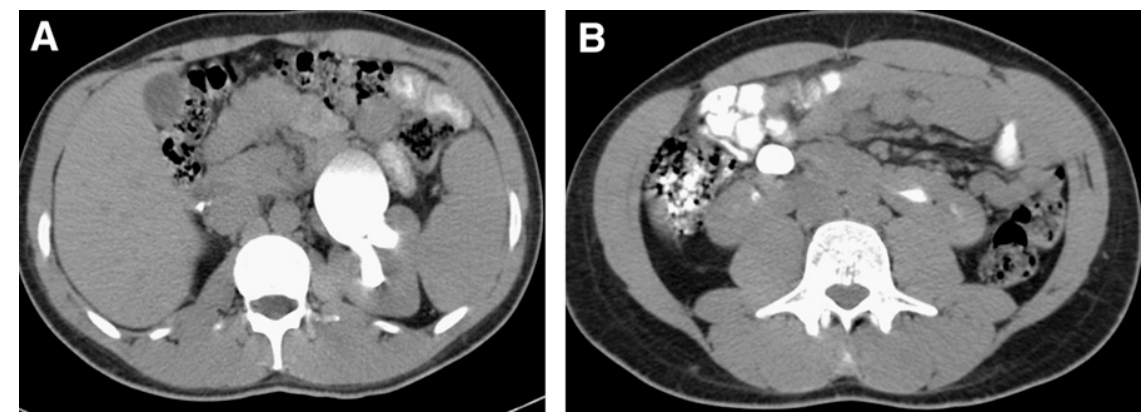

system. A standard imaging protocol was used because the nuclear medicine staff did not know the CT results at the time of the study and the patient did not relate a history of horseshoe kidney on initial questioning. The patient was hydrated well with intravenous fluid and then was given 99mTc-MAG3 intravenously. Single-detector dynamic posterior imaging was performed with the patient supine for 30 min and revealed asymmetric and progressive accumulation and retention of radiotracer in the left collecting system (Fig. 2A). The right collecting system cleared normally. Intravenous furosemide was then administered, and additional dynamic posterior imaging was performed for $30 \mathrm{~min}$ after infusion. Overall, this initial study demonstrated asymmetric perfusion and function, with significant anatomic dilation of the left extrarenal pelvis and marked retention of radiotracer in the left collecting system after diuretic challenge (Fig. 2B). Computer time-activity curves confirmed this visual assessment; a half-time emptying of 14 min represented an equivocal response.

The referring urologist believed that the patient's symptoms did not correlate with the degree of obstruction suggested by the scan; therefore, after consultation with the
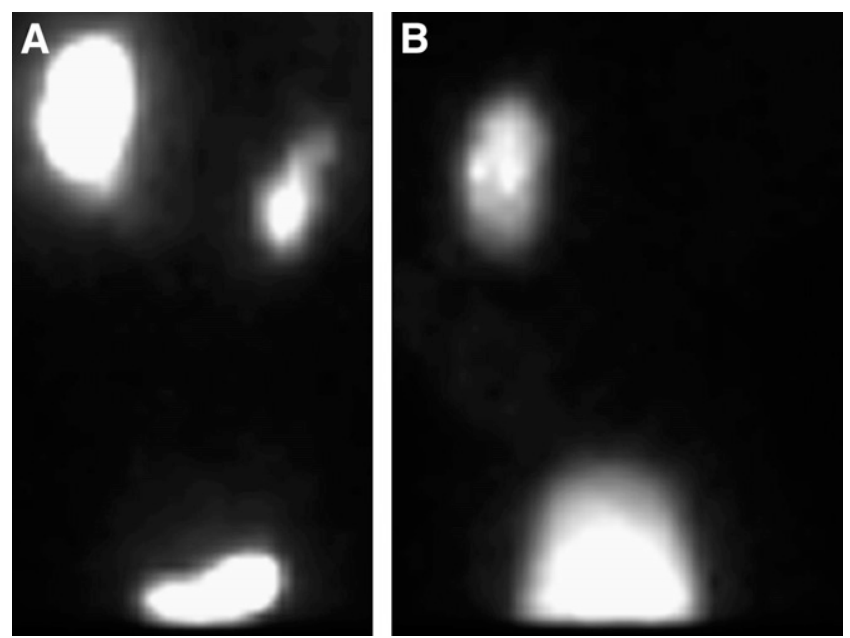

FIGURE 2. Study 1. (A) Dynamic prefurosemide posterior image at $28 \mathrm{~min}$ demonstrates asymmetric radiotracer accumulation and retention in left proximal collecting system. (B) Dynamic postfurosemide posterior image at $28 \mathrm{~min}$ demonstrates continued marked radiotracer retention. nuclear medicine staff, renal scintigraphy was repeated $8 \mathrm{~d}$ later with a modified protocol using a tailored approach given the known presence of a horseshoe kidney. The patient was hydrated well with intravenous fluid and then was given ${ }^{99 m}$ Tc-MAG3 intravenously. Dynamic imaging was performed for $30 \mathrm{~min}$, this time using dual-detector acquisition of both anterior and posterior data. Thereafter, supine (pregravity) static images of the horseshoe kidney were obtained in both anterior and posterior projections. The patient was then asked to ambulate for $15 \mathrm{~min}$ and void, after which another set of supine (postgravity) anterior and posterior images was acquired. Subsequently, at approximately $50 \mathrm{~min}$ after injection, intravenous furosemide was administered and dual-detector dynamic imaging in both anterior and posterior projections was performed for an additional $30 \mathrm{~min}$.

This second study showed that parenchymal perfusion and uptake, incorporating both anterior and posterior imaging data, were prompt and relatively symmetric. The split relative renal function for the left and the right moieties was $50 \% / 50 \%$ when calculated as a geometric mean. Persistent retention in the left collecting system was again identified on the baseline study (Figs. 3A and 3B). The use of preand postgravity static imaging with ambulation and voiding resulted in an additional $24 \%$ clearance of radiotracer from the anatomically dilated left collecting system (Figs. 3C through 3F). Subsequent imaging after diuretic administration resulted in near-complete emptying of the left collecting system (Fig. 4); computer time-activity curves were normal, and half-time emptying was $9 \mathrm{~min}$. Vicarious excretion of radiotracer into the hepatobiliary system was noted incidentally. On the basis of this follow-up study and the absence of symptom progression, the patient was followed conservatively by the referring urologist and no pyeloplasty was planned.

\section{DISCUSSION}

This case is of interest for several reasons. First, it demonstrates that although diuresis scintigraphy has proven useful in elucidating urinary tract obstruction, there are cases in which it fails to do so. Much work has been done to optimize the diagnostic value of renal scintigraphy, including altering the timing of diuretic injection (7) and using postdiuretic gravity-assisted drainage in infants and children (8). The topic of patient positioning was introduced by 

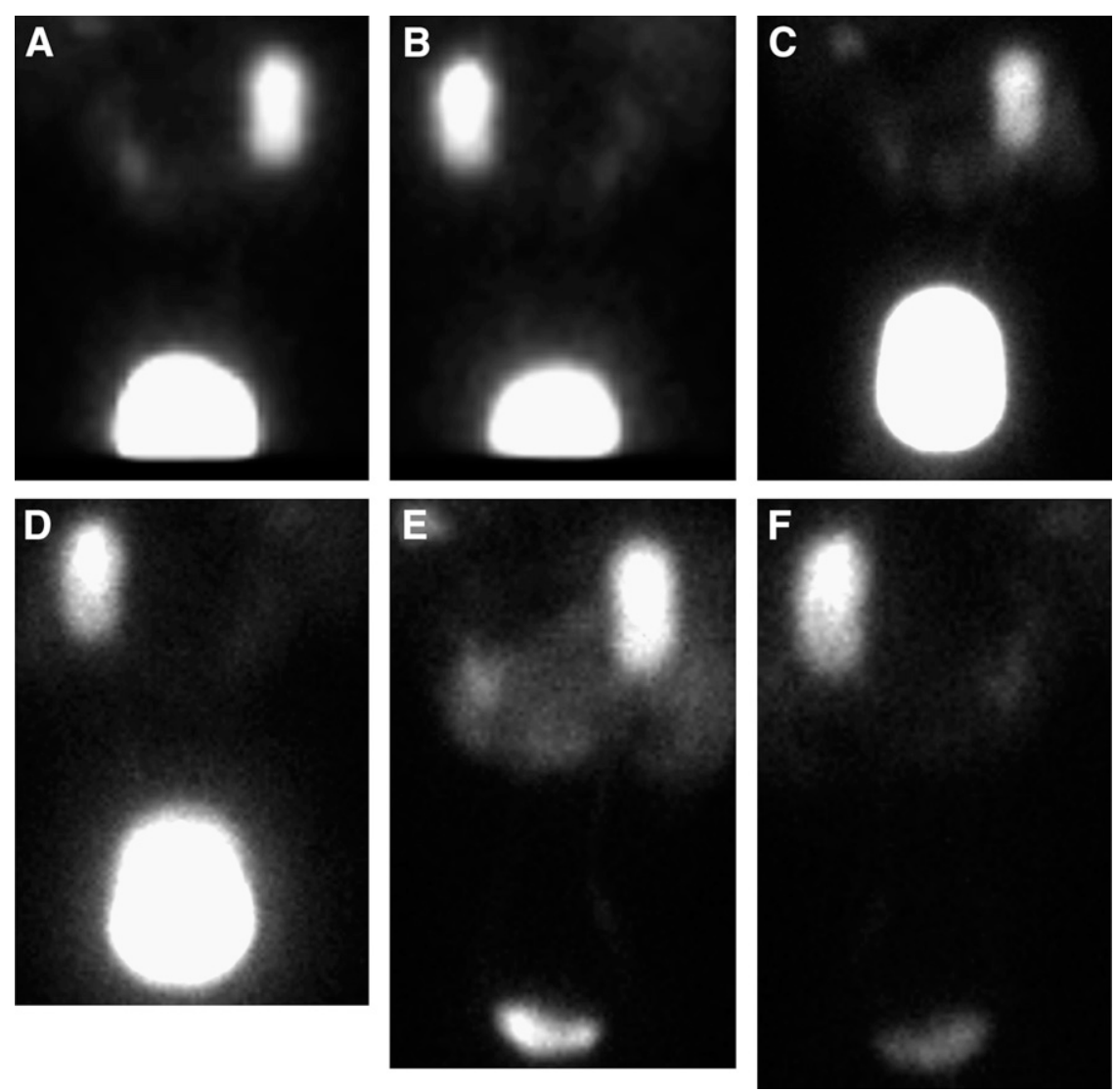

FIGURE 3. Study 2. (A and B) Dynamic prefurosemide anterior and posterior images at $28 \mathrm{~min}$ demonstrate findings similar to those of study 1 . (C and D) Static pregravity anterior (C) and posterior (D) images were obtained. After 15 min of ambulation, patient voided. Subsequent static postgravity anterior (E) and posterior $(F)$ images visually appear similar to pregravity images; however, computer region-of-interest activity measurements demonstrated additional $24 \%$ radiotracer clearance. an International Consensus Committee appointed by the Ninth International Symposium on Radionuclides in Nephrourology; unfortunately, the committee restricted comments on patient positioning to suggesting that the patient sit erect to elicit normal hydrostatic effects on urine flow, lie supine to prevent movement or syncope, and alternate between these 2 positions $(9,10)$.
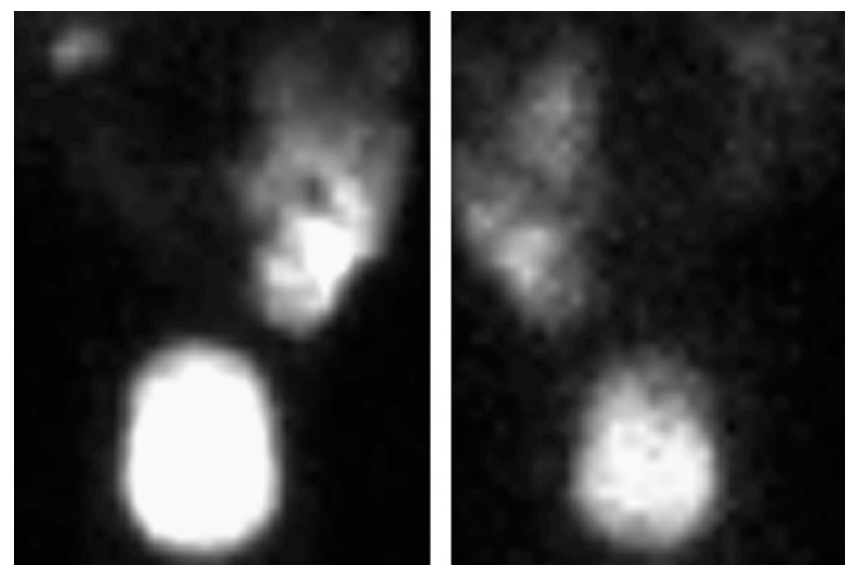

FIGURE 4. Study 2. Two different dynamic postfurosemide anterior and posterior images at $28 \mathrm{~min}$ after ambulation demonstrate nearly complete emptying of left renal collecting system. Half-time for emptying was 9 min. Incidentally noted was vicarious hepatobiliary excretion of radiotracer into small bowel.
However, in some patients, scintigraphic evaluation may be optimized by the use of simple maneuvers representing physiologic challenges that can be performed before diuretic administration and may result in more accurate evaluation of urinary flow dynamics. This is of particular importance in patients with congenital renal anomalies such as horseshoe kidney, for which altered positioning and urinary flow may under- or overestimate renal functional and excretory capabilities when only standard techniques are used. Simple physiologic challenges such as were used in this case (upright posture with ambulation, voiding) in conjunction with imaging in both anterior and posterior projections resulted in a more accurate assessment of renal function in this patient and should be considered in all cases of suspected retention in the renal collecting system. The importance of anterior and posterior dualdetector imaging in the scintigraphic evaluation of the horseshoe kidney is due to the collecting system being located in a more anteromedial position, and therefore imaging and analysis using routine posterior acquisition may result in mischaracterization of renal function. In any case in which a horseshoe or ectopic kidney is present or incidentally detected on the initial posterior dynamic study, anterior imaging should be incorporated before study completion. In the case presented here, an initial diuresis renogram using only posterior, supine imaging suggested obstruction. However, after physiologic challenge, and 
using anterior and posterior imaging, relative renal function and half-time emptying were obtained that offered compelling evidence that the collecting system was, in fact, unobstructed. Furthermore, supine imaging after physiologic challenge may prove to be a reasonable alternative to imaging while the patient is sitting upright, as suggested by the SNM Practice Guideline for the Performance of Pediatric Renal Scintigraphy (5), because such supine imaging is better tolerated by the patients and may reduce the incidence of motion artifacts.

\section{CONCLUSION}

The standardization and use of physiologic challenges during renal scintigraphy may prove to be an important adjunct to the traditional diuretic challenge and might be considered in patients in whom furosemide is contraindicated or whose postfurosemide imaging remains equivocal. The use of physiologic maneuvers, especially pregravity and postgravity images, is a vital supplement to diuretic challenge and may reduce the incidence of unnecessary surgery and follow-up imaging.

\section{REFERENCES}

1. Bauer SB. Anomalies of the upper urinary tract. In: Walsh PC, Retik AB, Vaughan ED Jr, et al., eds. Campbell's Urology. 8th ed. Philadelphia, PA: W.B. Saunders Co., 2002:1903-1906.

2. Domenech-Mateu JM, Gonzalez-Compta X. Horseshoe kidney: a new theory on its embryogenesis based on the study of a 16-mm human embryo. Anat Rec. 1988;222:408-417.

3. O'Reilly PH, Testa HJ, Lawson RS, et al. Diuresis renography in equivocal urinary tract obstruction. Br J Urol. 1978;50:76-80.

4. Lupton EW, Testa HJ, O'Reilly PH, et al. Diuresis renography and morphology in upper urinary tract obstruction. Br J Urol. 1979;51:10-14.

5. Mandell GA, Cooper JA, Leonard JC, et al. Society of Nuclear Medicine Procedure Guideline for Diuretic Renography in Children. Version 2. Available at: http://interactive.snm.org/docs/pg_ch29_0403.pdf. Accessed September 17, 2007

6. O'Reilly P, Aurell M, Britton K, et al. Consensus on diuresis renography for investigating the dilated upper urinary tract. J Nucl Med. 1996;37:1872-1876.

7. Türkölmez Ş, Atasever T, Türkölmez K, et al. Comparison of three different diuretic renal scintigraphy protocols in patients with dilated upper urinary tracts. Clin Nucl Med. 2004;29:154-160.

8. Wong DC, Rossleigh MA, Farnsworth RH. Diuretic renography with the addition of quantitative gravity-assisted drainage in infants and children. $J$ Nucl Med. 2000;41:1030-1036.

9. Conway J. Well-tempered diuresis renography: its historical development, physiological and technical pitfalls, and standardized technique protocol. Semin Nucl Med. 1992;22:74-84.

10. Kabala JE, Robinson PJA, Whittlestone T, et al. The urogenital tract: anatomy and investigation. In: Sutton D, ed. Textbook of Radiology and Imaging. 7th ed. Edinburgh, U.K.: Churchill Livingstone; 2003:912-917. 